\title{
Synthesis of an Ion Exchange Membrane by Radiation-Induced Grafting of Acrylic Acid onto Poly(tetrafluoroethylene)
}

\author{
Isao IshigaKI, Naotaka KamiYa, Takanobu Sugo, and Sueo MACHI \\ Japan Atomic Energy Research Institute, Takasaki Radiation Chemistry \\ Research Establishment, Watanuki, Takasaki, \\ Gunma 370-12, Japan.
}

(Received December 13, 1977)

\begin{abstract}
Acrylic acid was grafted onto a $100-\mu \mathrm{m}$ thick poly(tetrafluoroethylene) (PTFE) film by simultaneous irradiation method. The effect of the dose rate on the grafting and, the changes in the film thickness, water absorption, and electric resistance of the grafted film were investigated. The results suggested that the nongrafted layer remains in the middle part of the film at lower percent of grafting and then disappears as the grafting proceeds. Interference microscopy of the grafted film revealed that this grafting occurs by a gradual diffusion of the monomer through the successive grafted layers which swell in the reaction medium, i.e., the grafted front moves inward into film.
\end{abstract}

KEY WORDS Ion Exchange Membrane / Radiation / $\gamma$-Ray / Graft Polymerization / Acrylic Acid / Poly(tetrafluoroethylene) / Electric Resistance / Interference Microscopy /

Many theoretical studies and practical applications of ion-exchange membrane have been reported and its practical use in electrolytic systems has been carried out, since Juda, et al., ${ }^{1}$ reported on the characteristics of the resinous sulfonic cation exchanger. Particularly, many efforts have been devoted mainly to the development of homogeneous membranes based on the copolymers of styrene-divinyl benzene system. Those membranes are now used practically in the electrodialysis of blackish water and sea water to remove or to concentrate the solute.

Ion-exchange membranes are necessary for such properties as sufficient mechanical strength, chemical stability, and selective permeability of ions. The demands upon the polymer components in homogeneous membranes limit the full exploitation of either the membrane strength or the chemical and electrochemical properties. The incorporation of two distinct polymer components in the membrane structure has a certain advantage. The one component can provide a mechanically and chemically stable membrane matrix and the other component serves as a transport.

Poly(tetrafluoroethylene)(PTFE) is well-known as one of the most excellent materials for chemical resistance and mechanical strength. One can expect to obtain an excellent ion-exchange membrane by the combination of PTFE with components having electrolyte groups. From this view point, the authors have studied the radiationinduced graft copolymerization of acrylic acid onto PTFE film.

By using simultaneous or post irradiation grafting techniques, PTFE was found to be grafted by various monomers such as vinyl acetate, ${ }^{2}$ styrene, ${ }^{3-7} \mathrm{~N}$-vinylpyrrolidone, ${ }^{8-10}$ acrylic acid, ${ }^{6,11,12}$ 4-vinyl pyridine, ${ }^{8,12-14}$ methyl methacrylate. $^{3} \quad$ The grafting is largely affected by the diffusion of a monomer into the PTFE matrix which scarcely swells in any solvent or monomer. Restaino, et al., ${ }^{2}$ studied kinetically the gamma ray-induced grafting of vinyl acetate to PTFE film and found that vinyl acetate grafts only on the film surface. However, for grafting of styrene and methyl methacrylate onto PTFE, Chapiro ${ }^{3}$ found that if the grafting is carried out under well-defined conditions in which the graft copolymer swells, the grafting can proceed progressively into the PTFE matrix and finally a homogeneous 
graft copolymer is obtained.

Dobo, et al., ${ }^{5}$ determined the penetration of grafted styrene into pre-irradiated PTFE by measuring the optical density across microsections of the grafted and colored films and found that grafting begins at the surface and then takes place in a relatively narrow travelling front into the polymer matrix.

Chapiro, et al., who have studied extensively the grafting of acrylic acid ${ }^{6,11,12}$ and 4-vinyl pyridine $^{12,13}$ onto PTFE, obtained polyfunctional membranes by grafting two monomers in successive steps; these swell in water to a much greater extent than membranes on which was grafted either acrylic acid or vinyl pyridine alone. ${ }^{12,15}$ They also prepared the mosaic membrane grafted with those two monomers in the form of a grid ( $0.5-\mathrm{mm}$ wide line) by using a well-defined technique for shielding electron beams and found that the diffusion constant of ion pairs in the membrane thus obtained is $10^{2}$ to $10^{3}$ times higher than for carboxylic membranes with the same overall grafting ratio. ${ }^{16}$

By using radiation-induced grafting, it is of great interest not only to prepare an ion-exchange membrane with excellent properties but also to study the effects of the distribution of electrolytes grafted in the matrix on the physical and electrochemical properties. In the present work, the radiation-induced grafting of acrylic acid onto PTFE film in aqueous solution and the various properties of the membrane are studied.

\section{EXPERIMENTAL}

\section{Materials}

PTFE film (100- $\mu \mathrm{m}$ thickness; specific gravity, $2.2 \mathrm{~g} / \mathrm{cm}^{3}$ ) obtained commercially was washed with an aqueous solution of $15-w t \%$ neutral detergent for $24 \mathrm{hr}$, rinsed several times with acetone and distilled water, and dried in vacuo for $24 \mathrm{hr}$ at room temperature.

Reagent grade acrylic acid was passed through a column of ion-exchange resin (Amberlyst A-27, Rohm \& Haas Co. Ltd.) so as to be free from any polymerization inhibitor. The other chemicals were reagent grade and used without further purification.

\section{Graft Polymerization}

A glass ampoule containing strips $(6 \times 15 \mathrm{~cm})$ of PTFE was degassed for $30 \mathrm{~min}$ under reduced pressure less than $1 \times 10^{-3} \mathrm{mmHg}$. The monomer solution, deaerated by bubling nitrogen, was introduced into the evacuated ampoule. The sample thus obtained was subjected to $\gamma$-ray irradiation from Co-60. The graft polymerization was carried out in a nitrogen atmosphere. After the irradiation, the films were taken out, washed and soaked overnight in distilled water to eliminate the residual monomer and homopolymer contained in the film. It was ascertained that the homopolymer was not extracted futher additional soaking. The film was then dried under vacuum for $24 \mathrm{hr}$ at room temperature and weighed.

The percent of grafting was calculated as

$$
\left(w_{\mathrm{g}}-w_{\mathrm{i}}\right) / w_{\mathrm{i}} \times 100
$$

where $w_{\mathrm{i}}$ and $w_{\mathrm{g}}$ represent the weights of the initial and grafted film, respectively.

\section{Water Absorption}

The grafted film was immersed in distilled water for more than $24 \mathrm{hr}$ at room temperature. The film was taken out, quickly wiped with filter paper, and weighed. The percent absorption of water was calculated as

$$
\left(w_{\mathrm{s}}-w_{\mathrm{g}}\right) / w_{\mathrm{g}} \times 100
$$

where $w_{\mathrm{g}}$ and $w_{\mathrm{s}}$ represent the weights of dry and wet films, respectively.

\section{Electric Resistance}

The grafted film was immersed in $0.5-\mathrm{N} \mathrm{NaCl}$ solution for more than $24 \mathrm{hr}$ prior to the measurement of electric resistance. The film, swollen with $0.5-\mathrm{N} \mathrm{NaCl}$ solution, was cut into a disk (available area, $33.0 \mathrm{~cm}^{2}$ ) and mounted between the platinum electrodes in the electrolytic cell. The $0.5-N \mathrm{NaCl}$ solution filled on both sides of the film. The overall electric resistance between the electrodes was measured at $25^{\circ} \mathrm{C}$ using an ohmmeter (LCR Meter 4261A, YokogawaHewlett-Packard) working at $1000 \mathrm{~Hz}$. The electric resistance of the membrane $(R)$ was calculated using the equation

$$
R=\left(R_{\mathrm{m}+\mathrm{c}}-R_{\mathrm{c}}\right) \times S / d
$$

where $R_{\mathrm{m}+\mathrm{c}}$ and $R_{\mathrm{c}}$ represent apparent values with 
and without the membrane, and $S$ and $d$ represent the available area and thickness of the film swollen in $0.5-\mathrm{N} \mathrm{NaCl}$ solution.

\section{Grafting Distribution}

The grafting distribution perpendicular to the film surface was observed using an interference microscope (PERAVAL interphako 30-G310, Carl Zeiss JENA).

The film was sliced perpendicularily to its surface using a microtome (Large Sliding Microtome LS-113, Yamato Koki). The 20- $\mu$ m thick slice thus obtained was observed and photographed under an interference microscope.

\section{RESULTS AND DISCUSSION}

Since PTFE scarcely swells in any solvent, the effects of the monomer concentration and the solvent on the graft polymerization of acrylic acid onto PTFE film were first investigated as shown in Table I. It can be seen that the percent of

Table I. Effect of monomer concentration and solvent on the percent of grafting of acrylic acid onto $\mathrm{PTFE}^{\mathrm{a}}$

\begin{tabular}{clcc}
$\begin{array}{c}\text { Monomer } \\
\text { concn, } \\
\text { wt } \%\end{array}$ & Solvent & $\begin{array}{c}\text { Mohr's } \\
\text { salt, } \\
\text { wt } \%\end{array}$ & $\begin{array}{c}\text { Percent } \\
\text { grafting, } \\
\%\end{array}$ \\
\hline 10 & Water & 0.19 & 7.38 \\
30 & Water & 0.19 & 24.0 \\
50 & Water & 0.19 & 23.5 \\
70 & Water & 0.19 & 28.6 \\
30 & Acetone & - & 0.20 \\
30 & Toluene & - & 2.13 \\
\hline
\end{tabular}

a Polymerization conditions: dose rate, $2.7 \times 10^{4}$ $\mathrm{rad} / \mathrm{hr}$; irradiation temp, room temp; irradiation time, $16 \mathrm{hr}$; film thickness, $100 \mu \mathrm{m}$.

grafting obtained by using water as the solvent is not remarkedly affected at the monomer concentration more than $30 \mathrm{wt} \%$, and that the highest one is obtained in water among other organic solvents. Poly(acrylic acid) can be dissolved in water so that the grafted PTFE swells in water as is described below. Although poly(acrylic acid) dissolves neither in toluene nor acetone, the acrylic acid-grafted PTFE film swells slightly in these two solvents. In fact, the percents of swelling of the film having the percent of grafting of $24.0 \%$ were determined as $2.5 \%$ in toluene and
$1.5 \%$ in acetone from the weight increase of the film after soaking in the solvent for $48 \mathrm{hr}$ at room temperature. The percent of grafting obtained in toluene solution, as shown in Table I, was also found to be larger than that in acetone solution. This is attributable to the difference in swelling of the grafted zone, since the grafting of PTFE begins at the surface and then proceeds progressively into the matrix by the diffusion of the monomer through the grafted zone.

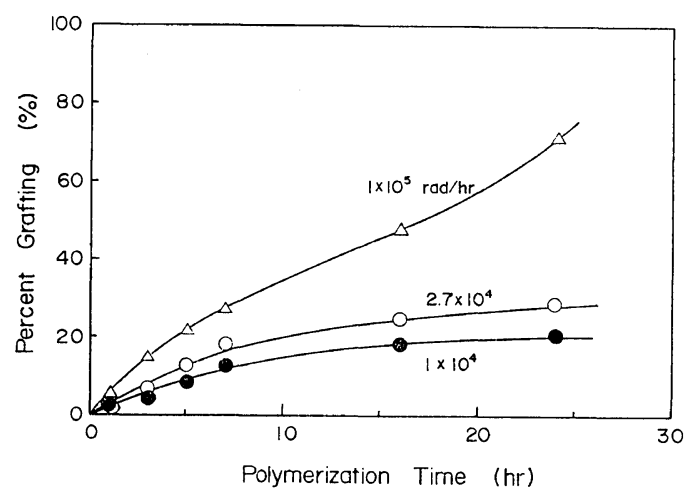

Figure 1. The percent of grafting against polymerization time: acrylic acid, $50 \mathrm{wt} \%$; solvent, $\mathrm{H}_{2} \mathrm{O}$; polymn. temp, room temp; film thickness, $100 \mu \mathrm{m}$.

Figure 1 shows the percent of grafting against the irradiation time. In comparison with the original film, the appearance of the grafted film was not markedly changed irrespective of the percent of grafting and the dose rate, but the wettability of the film surface with water increased remarkably with increasing the percent of grafting. Since, as mentioned above, PTFE dose not swell in an aqueous solution of acrylic acid, the grafting seems to proceed from the surface to the inside of the film along with diffusion of the acrylic acid through the matrix which comes to swell in the monomer solution due to the grafting. However, as seen in Figure 1, the graft polymerization was not completely governed by the diffusion of acrylic acid into PTFE matrix. The grafting rate was found to increase with the dose rate. If the graft polymerization were predominantly dependent only on the diffusion of monomer into the matrix, the rate of graft polymerization should be constant irrespective of the dose rate.

The initial rates of grafting, determined from the grafting-time curves in Figure 1, were plotted 


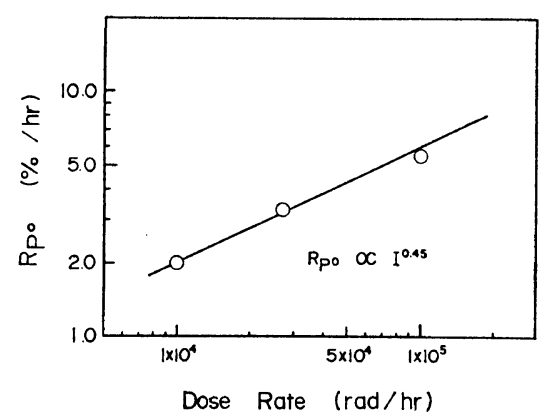

Figure 2. Logarithmic plots of initial grafting rate $\left(R_{\mathrm{p}_{0}}\right)$ against dose rate: acrylic acid, $50 \mathrm{wt} \%$; solvent, $\mathrm{H}_{2} \mathrm{O}$; polymn. temp, room temp; film thickness, 100 $\mu \mathrm{m}$.

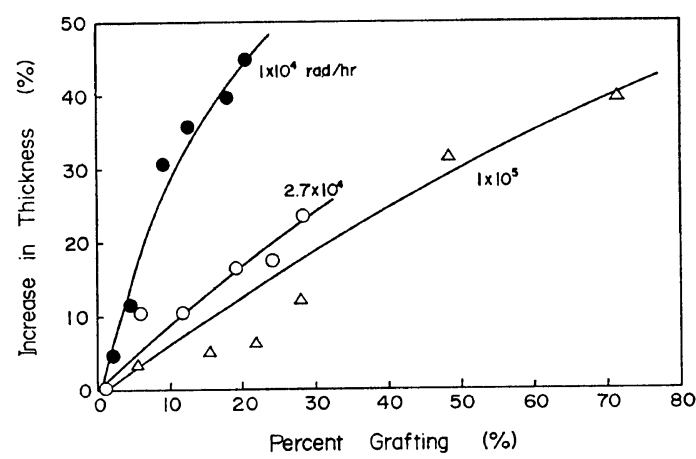

Figure 3. Increase in the thickness of grafted film against the percent of grafting: acrylic acid, $50 \mathrm{wt} \%$; solvent, $\mathrm{H}_{2} \mathrm{O}$; polymn. temp, room temp; film thickness, $100 \mu \mathrm{m}$.

against the dose rate shown in Figure 2. The initial rate is proportional to a 0.5 power of dose rate.

The poly(tetrafluoroethylene) underwent dimensional changes upon grafting. The increase in thickness at various dose rates was shown in Figure 3 as a function of the percent of grafting of acrylic acid. At the same percent grafting, the lower the dose rate, the larger the increase in thickness. This result presumably indicates that the grafting proceeds to a deeper region from the film surface at a lower dose rate than at a higher dose rate. In other words, at a higher dose rate, the grafting seems to be localized in the region near the surface.

Figure 4 shows the changes in three-dimensional directions. As described already, the $100 \mu \mathrm{m} \times$

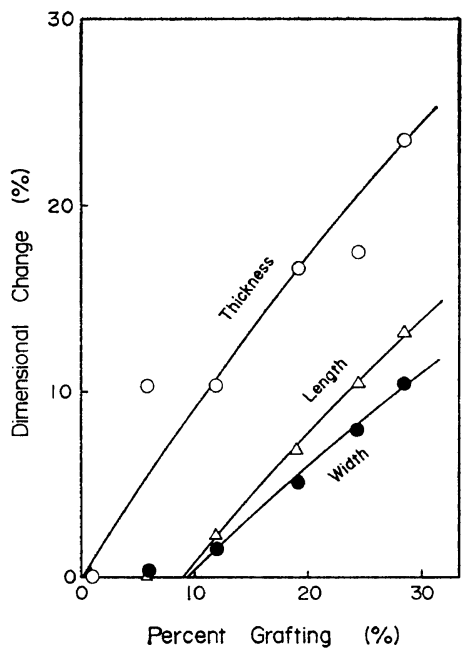

Figure 4. Dimensional changes of grafted film against the percent of grafting: acrylic acid, $50 \mathrm{wt} \%$; solvent, $\mathrm{H}_{2} \mathrm{O}$; dose rate, $2.7 \times 10^{4} \mathrm{rad} / \mathrm{hr}$; polymn. temp, room temp; original film thickness, $100 \mu \mathrm{m}$.

$6 \mathrm{~cm} \times 15 \mathrm{~cm}$ strips of film were used in this work. The dimensional changes in length $(15 \mathrm{~cm})$ and in width $(6 \mathrm{~cm})$ are considerably different from that in thickness. Although the thickness increases proportionally with the percent of grafting, the length and width of the film do not change within $10 \%$ grafting and then increase. From this result, it is supposed that, at a lower grafting, the nongrafted layer remains in the middle layer of the film and prevents dimensional changes in directions of length and width but not in thickness of the film, even at a lower grafting less than $10 \%$. As will be discussed later, the nongrafted layer in the film disappeared as the graft polymerization proceeded more than $\mathrm{ca} .10 \%$ grafting under the experimental conditions.

In general, polymer grafted by hydrophilic monomer swells in water or absorbs water. The water absorption of the acrylic acid-grafted film is shown as a function of the percent of grafting in Figure 5. The water absorption was found to increase with an increasing percent in the grafting and to be higher in the film grafted at a lower dose rate. The graft at a lower dose rate gives a more homogeneous distribution of the acrylic acid in polymer matrix as compared with that at a higher dose rate. The dose rate dependency of the water absorption coincided with that of the increase in 


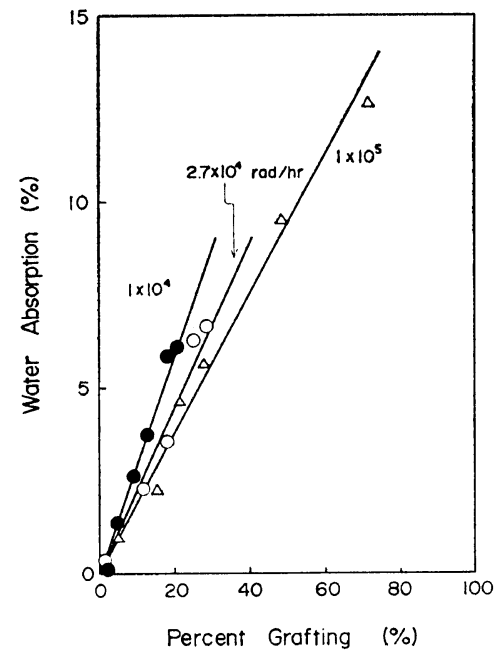

Figure 5. Water absorption of grafted film against the percent of grafting: acrylic acid, $50 \mathrm{wt} \%$; solvent, $\mathrm{H}_{2} \mathrm{O}$; polymn. temp, room temp; original film thickness, $100 \mu \mathrm{m}$.

thickness by grafting (see in Figure 3).

Figure 6 shows the relationship between the electric resistance and the percent of grafting of the films obtained at different dose rate. The electric resistances were found to decrease as the grafting percent increases and to fall sharply at a certain percent of grafting $(10-20 \%)$ which increases with the dose rate. This result can be explained by the progressive grafting mechanism from the surface to the center of the film. In the region where the film gives a higher electric

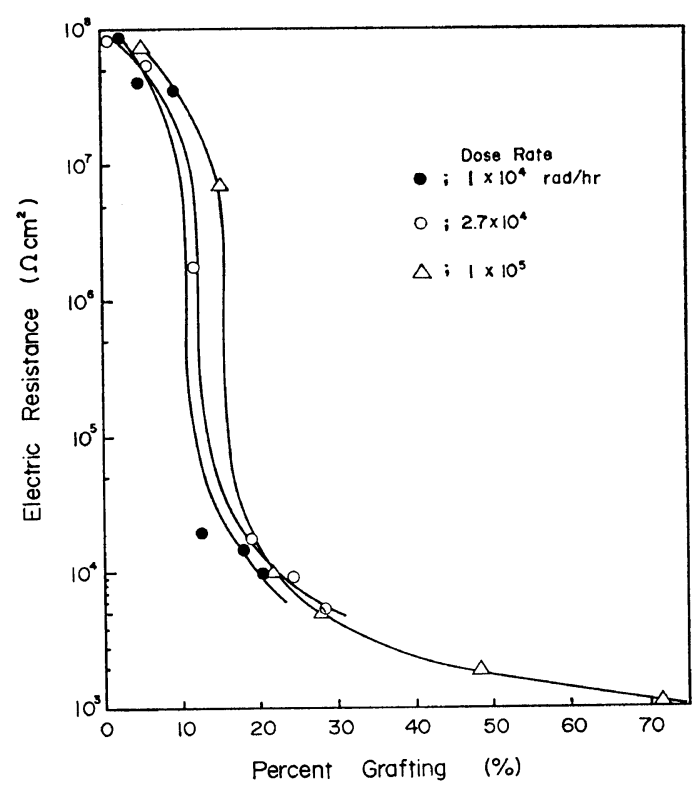

Figure 6. Electric resistance of grafted film against the percent of grafting: acrylic acid, $50 \mathrm{wt} \%$; solvent, $\mathrm{H}_{2} \mathrm{O}$; Polymn. temp, room temp; original film thickness, $100 \mu \mathrm{m}$.

resistance, the grafting front does not reach the center of the film; i.e., the nongrafted layer with low electric conductivity remains. The abrupt decrease in the electric resistance of the film at a grafting percent more than $10-20 \%$ undoubtedly indicates the disappearance of the nongrafted layer in the film. This means that the grafting front reaches the center of film. The dose rate

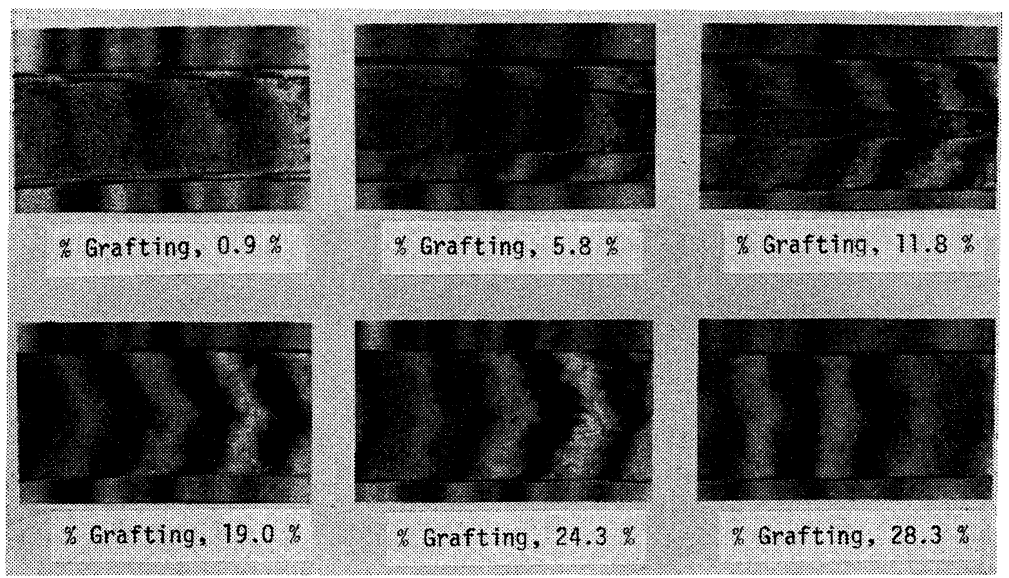

Photo 1. Interference micrograph of grafted film: acrylic acid, $50 \mathrm{wt} \%$; solvent, $\mathrm{H}_{2} \mathrm{O}$; dose rate, $2.7 \times 10^{4} \mathrm{rad} / \mathrm{hr}$; polymn. temp, room temp; original film thickness, $100 \mu \mathrm{m}$. 


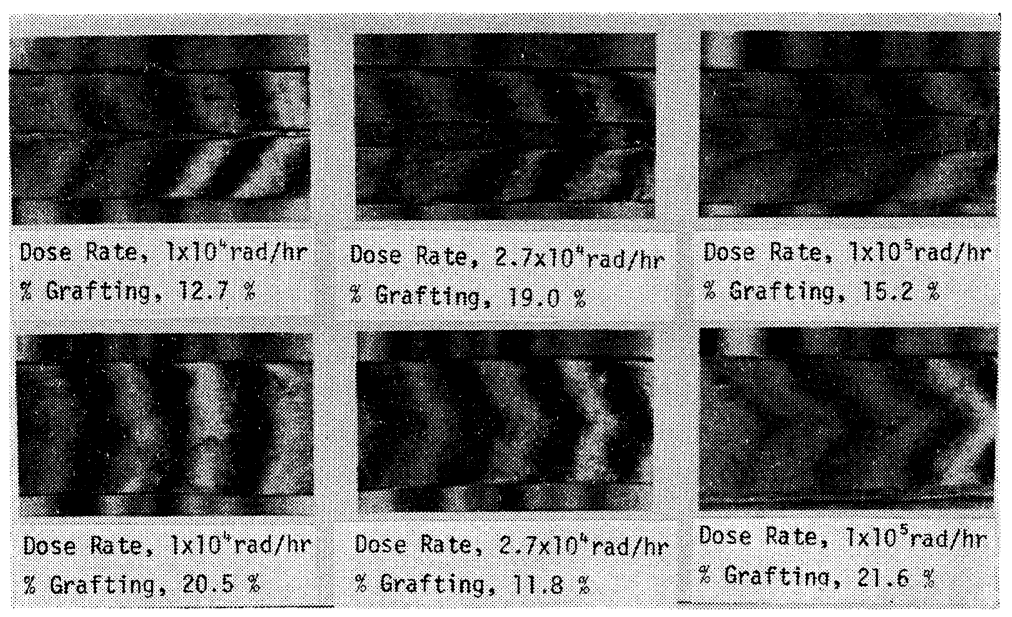

Photo 2. Interference micrograph of grafted film: acrylic acid, $50 \mathrm{wt} \%$; solvent, $\mathrm{H}_{2} \mathrm{O}$; polymn. temp, room temp; original film thickness, $100 \mu \mathrm{m}$.

dependency of the percent of grafting which brings about an abrupt change in electric resistance of film can be reasonably understand, because the lower the dose rate, the deeper the grafted layer at the same grafting percent as shown in Figure 3. This means the grafting front travels relatively faster at a lower dose rate. After falling abruptly, the electric resistances decrease gradually due to an increase in the amount of electrolyte(acrylic acid) and the uniformity of its distribution.

In order to elucidate the grafting distribution in the direction of film thickness, the cross section sliced perpendicularily to the film surface was observed using an interference microscope. Photo 1 shows the change in the grafting distributions for the grafted film obtained at a constant dose rate of $2.7 \times 10^{4} \mathrm{rad} / \mathrm{hr}$. It can be seen that the graft polymerization proceeds from the both surfaces to the middle part of the film. At a lower grafting less than $12 \%$, the nongrafted layer in the middle part of the film is clearly observed. After the nongrafted layer disappeared (more than $19 \%$ ), the slopes of the interference stripes decreased with an increase in the percent of grafting. This means that the grafting distribution becomes homogeneous as the graft proceeds.

The effects of dose rate on the thickness of nongrafted layer and on the grafting distribution are shown in Photo 2. The lower the dose rate for grafting, the thinner the nongrafting layer; i.e., as indicated above regarding the result in Figure
3 , the grafting proceeds more deeply at a low dose rate than it does at a high dose rate at the same percent of grafting. In addition, the slope of the interference stripes became smaller as the dose rate decreased. In other words, the distribution of grafting becomes more homogeneous at a lower dose rate.

Figure 7 shows the plots of the nongrafted layer thickness determined by the microscopy of the grafted film. It can be seen that the nongrafted layer thickness decreases with an increase in the percent of grafting for any dose rate, and that the percent of grafting at which the nongrafted layer disappears decreases with a decreasing

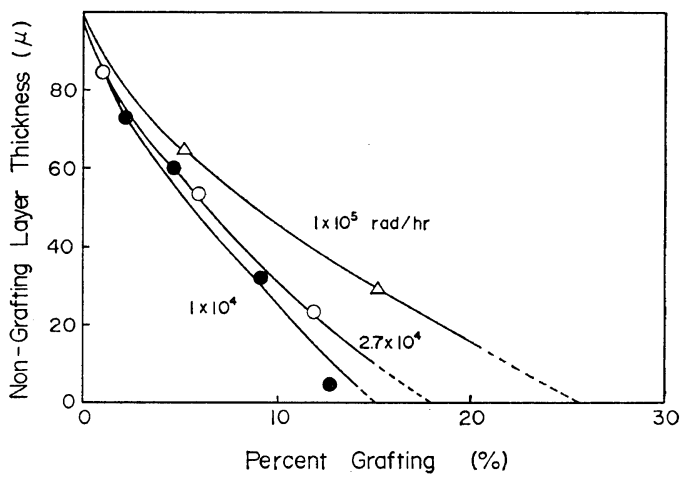

Figure 7. Non-grafted layer thickness of grafted film against the percent of grafting: acrylic acid, $50 \mathrm{wt} \%$; solvent, $\mathrm{H}_{2} \mathrm{O}$; polymn. temp, room temp; original film thickness, $100 \mu \mathrm{m}$. 
dose rate.

These results obtained by microscopy of the grafted films are in close agreement with those deduced from measurements of the change in thickness, water absorption, and electric resistance for the acrylic acid-grafted poly(tetrafluoroethylene) films.

\section{REFERENCES}

1. W. Juda and W. A. McRae, J. Am. Chem. Soc., 72, 1044 (1950).

2. A. J. Restaino and W. N. Reed, J. Polym. Sci., 36, 499 (1959).

3. A. Chapiro, J. Polym. Sci., 34, 481 (1959).

4. A. Chapiro and A. Matsumoto, J. Polym. Sci., 57, 743 (1962).

5. J. Dobó, A. Somogyi, and T. Czvikovszky, $J$. Polym. Sci., Part C, No. 4, 1173 (1964).

6. A. Chapiro, G. Derai, and A.-M. JendrychowskaBonamour, Eur. Polym. J., 7, 1595 (1971).
7. S. Munari, F. Vigo, M. Nicchia, and P. Canepa, J. Appl. Polym. Sci., 20, 243 (1976).

8. P. Aptel, J. Cuny, J. Jozefowicz, G. Morel, and J. Neel, J. Appl. Polym. Sci., 16, 1061 (1972).

9. P. Aptel, J. Cuny, J. Jozefowicz, G. Morel, and J. Neel, Eur. Polym. J., 9, 877 (1973).

10. A. Chapiro, A.-M. Jendrychowska-Bonamour, G. Morel, and R. Oppelt, Eur. Polym. J., 9, 847 (1973).

11. A. Chapiro and P. Seidler, Eur. Polym. J., 1, 189 (1965).

12. A. Chapiro, G. Bex, A.-M. JendrychowskaBonamour, and T. O’Neill, Adv. Chem. Ser., 91, 560 (1969).

13. A. Chapiro, E. Gordon, and A.-M. Jendrychowska-Bonamour, Eur. Polym. J., 9, 975 (1973).

14. G. Canepa, S. Munari, C. Rossi, and F. Vigo, Desalination, 13, 159 (1973).

15. A.-M. Jendrychowska-Bonamour, J. Chem. Phys., 70, 20 (1973).

16. A. Chapiro, Radiat. Phys. Chem., 9, 55 (1977). 